\title{
LES TECHNIQUES DE CONTRÔLE DES PASSES A POISSONS
}

\author{
F. TRAVADE* et M. LARINIER**
}

* EDF - Etudes et Recherches - 6, Quai Watier - 78401 CHATOU CEDEX

** CSP-CEMAGREF, GHAAPPE - Institut de Mécanique des Fluides - Avenue du Professeur Camille Soula - 31400 TOULOUSE

\section{INTÉRÊT DU CONTRÔLE DES PASSES A POISSONS}

Les opérations de suivi et de contrôle du fonctionnement des ouvrages de franchissement sont importantes à plusieurs titres : éventuelle,

- vérification de l'efficacité des ouvrages après leur mise en service et mise au point

- recueil d'informations techniques et biologiques indispensables à la conception et à l'optimisation des futurs ouvrages (retour d'expérience),

- connaissance des populations de poissons migrateurs et des caractéristiques de leurs migrations, nécessaire tant pour le dimensionnement d'éventuels ouvrages à construire sur le même cours d'eau en amont que pour la gestion rationnelle des populations.

Les techniques à mettre en oeuvre se regroupent sous plusieurs rubriques :

- contrôle du fonctionnement hydraulique et mécanique de la passe,

- recueil d'informations biologiques qualitatives indicatrices de la réalité du franchissement,

- comptage des poissons transitant dans les passes,

- relativisation des passages par rapport à la population migrante, qui représente en fait l'efficacité réelle de la passe.

\section{CONTRÔLE DU FONCTIONNEMENT HYDRAULIQUE ET MÉCANIQUE}

II s'agit là soit de mesures simples, soit d'observations réalisées lors de la mise en service puis à intervalles réguliers, et destinées à s'assurer de la conformité de la passe aux critères définis lors de la conception du dispositif.

\subsection{Paramètres hydrauliques}

On procèdera à la mesure des niveaux en divers points singuliers de l'ouvrage (amont, aval, bassins particuliers...) et , pour les passes à bassins, à celles des niveaux et des chutes pour chacun des bassins ainsi qu'à l'entrée de l'ouvrage. Ces mesures sont à réaliser dans plusieurs configurations de niveaux amont et aval.

On s'assurera que la structure des écoulements et le niveau d'agitation en divers points de la passe restent compatibles avec les exigences particulières des diverses espèces : jets plongeants ou de surface au droit des chutes, jet "vrillé", présence de courants de retour dans les bassins...

\subsection{Paramètres mécaniques}

Pour les passes à bassins ou à ralentisseurs, les contrôles porteront sur le fonctionnement des divers organes de régulation du débit ou de la chute (vannes asservies). Pour les passes 
"mécaniques" (ascenseurs, écluses) on contrôlera l'ensemble des mécanismes assurant le déroulement du cycle de fonctionnement. On vérifiera la durée de chacune des phases du cycle.

\subsection{Obstruction et colmatage de la passe}

Une attention particulière devra être apportée aux éventuelles obstructions par des corps dérivants qui peuvent, dans certaines zones sensibles (pertuis entre bassins, sortie de la passe, grilles d'injection des débits complémentaires d'appoint), gêner notablement le passage des poissons ou réduire l'attractivité de la passe sans se traduire obligatoirement par une perturbation hydraulique très visible. A cet égard, les orifices noyés sont à contrôler très attentivement.

\section{RECUEIL D'INFORMATIONS BIOLOGIQUES QUALITATIVES OU SEMI-QUANTITATIVES}

En dehors de toute approche quantitative de l'efficacité d'un ouvrage, il existe un certain nombre d'informations indirectes qui peuvent indiquer si le fonctionnement de l'ouvrage est globalement satisfaisant ou non. Ces informations sont d'autant plus intéressantes s'il est possible de les recueillir également avant l'installation du dispositif de franchissement sur l'obstacle :

- observation de poissons remontant dans la passe,

- fréquence des sauts de migrateurs au pied de l'obstacle ou densité des poissons s'ils sont visibles,

— densité des pêcheurs à l'aval de l'obstacle (excellent indicateur d'un blocage des poissons!),

- indices d'abondance des migrateurs (observations, captures) et comptage des frayères (nids de ponte pour les salmonidés et les lamproies, "bull" pour les aloses) en amont de la passe.

Cette liste n'est pas exhaustive, et un certain nombre d'autres sources de renseignements peuvent servir à cette appréciation qualitative du fonctionnement d'une passe.

\section{COMPTAGE DES POISSONS DANS LES PASSES}

\subsection{Piégeage}

Dans son principe, le comptage par piégeage consiste à capturer les poissons dans un dispositif approprié installé dans la passe ou à sa sortie et à en effectuer manuellement le dénombrement avant de les relâcher à l'amont.

Le piège est généralement constitué d'une cage grillagée ou d'une chambre munie d'un système anti-retour (principe de la nasse) disposée sur le trajet du poisson. La récupération des poissons après capture fait appel à des systèmes très variés plus ou moins automatisés incluant la vidange partielle de la chambre de capture, le relevage, par un dispositif manuel ou électrique, de l'ensemble de la cage de capture, d'un fond mobile grillagé permettant d'isoler les poissons dans une faible épaisseur d'eau, ou d'une cuve disposée sur le fond de la chambre (Fig. 1).

Le passage des poissons à l'amont peut se faire soit avec manipulation du poisson (épuisette, tube PVC), soit par déversement direct (vidange d'une cuve par gravité), soit par ouverture de grilles autorisant l'échappement du poisson vers l'amont.

Le dimensionnement du piège doit prendre en compte le nombre maximal instantané de poissons susceptibles d'être présents dans l'installation. II sera donc fonction des pics journaliers de migration des différentes espèces et de la fréquence des contrôles. Le volume du piège est calculé en prenant en compte un volume minimal de l'ordre de 15 litres/kg de poisson stabulé (CLAY, 1961; BELL, 1986).

Les dimensions les plus courantes des pièges rencontrés en France sont de $2.5 \mathrm{~m}$ à $4.5 \mathrm{~m}$ pour la longueur, de $1.5 \mathrm{~m}$ à $3.0 \mathrm{~m}$ pour la largeur, et de $1 \mathrm{~m}$ à plus de 2.5 mètres pour la profondeur. 
Les opérations de comptage doivent être effectuées à intervalles réguliers (en général une à plusieurs fois par jour) pour éviter la stabulation prolongée des poissons. Une surveillance pour prévenir les phénomènes de colmatage et le braconnage est par ailleurs nécessaire.

Les coûts d'un dispositif de piégeage sont très variables suivant la taille du dispositif de franchissement, la sophistication du système de manipulation et la nature des espèces migratrices concernées. Ils peuvent varier de moins de $10000 \mathrm{~F}$ à $150000 \mathrm{~F}$, correspondant à $1 \%$ à $10 \%$ du coût de la passe. Les coûts de maintenance et de gestion de ces ouvrages sont en général assez élevés, essentiellement du fait de la main-d'oeuvre nécessaire aux opérations. Ils sont très variables d'un site à l'autre suivant l'abondance et la nature des migrateurs, le niveau d'automatisation du piège et la lourdeur - souvent imprévisible - des opérations d'entretien et de nettoyage des grilles du dispositif. Suivant les sites, de 0.5 à 4 personnes sont nécessaires à la gestion des pièges durant la ou les périodes de fonctionnement.

Le principal avantage de ce type de contrôle est une mise en oeuvre relativement aisée, notamment pour les ouvrages de petite taille. Les autres avantages résident dans la fiabilité de détermination des espèces, la possibilité de recueil des caractéristiques biologiques des poissons (taille, poids, sexe, etc...) et de prélèvement d'individus pour procéder à leur marquage ou pour leur utilisation comme géniteur de repeuplement.

Les inconvénients sont les risques de blessures ou de traumatismes (voire de mortalité sur certaines espèces fragiles comme l'alose), la lourdeur de la maintenance et des besoins en personnel sur les ouvrages situés sur les grands cours d'eau, ainsi que l'impossibilité de recueillir des données en continu. Un autre inconvénient, difficile à apprécier, est l'impact souvent négatif de l'ouvrage de piégeage sur le fonctionnement de la passe, certaines espèces, comme l'alose, se montrant particulièrement méfiantes à s'engager dans un piège.

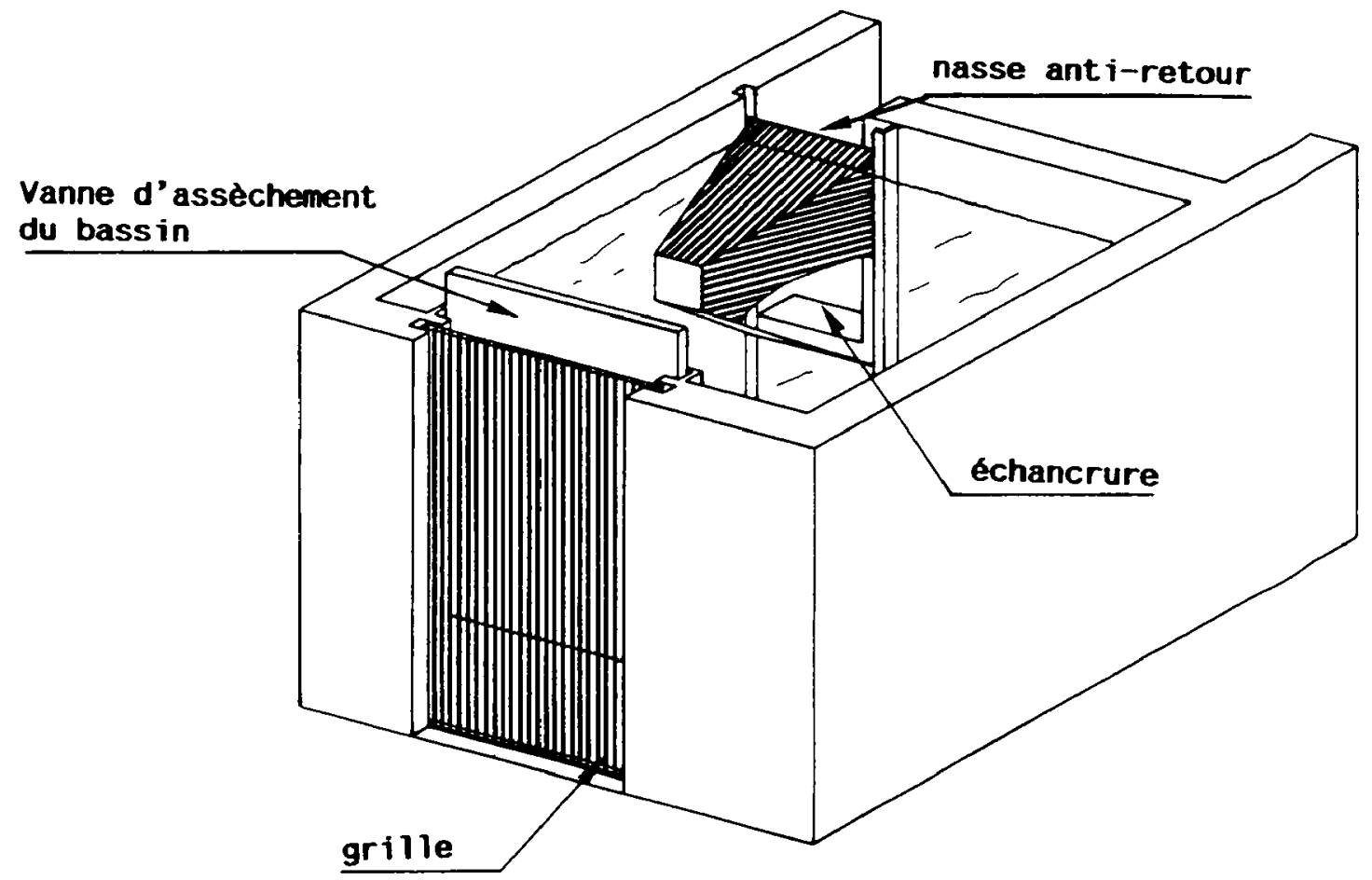

Figure 1 : Dispositif de piégeage installé dans une petite passe à bassins. Le poisson est récupéré manuellement par vidange du bassin.

Figure 1 : Trapping device used to count fish in small pool fishways. Fish is manually caught after emptying the pool. 


\subsection{Compteur automatique a résistivité}

Le principe du compteur à résistivité est basé sur la différence de conductivité entre l'eau et le corps du poisson : on oblige le poisson à franchir une série de trois électrodes immergées constituant deux branches d'un pont de Wheastone alimenté en courant alternatif, les deux autres branches étant formées de résistances fixes (Fig. 2).

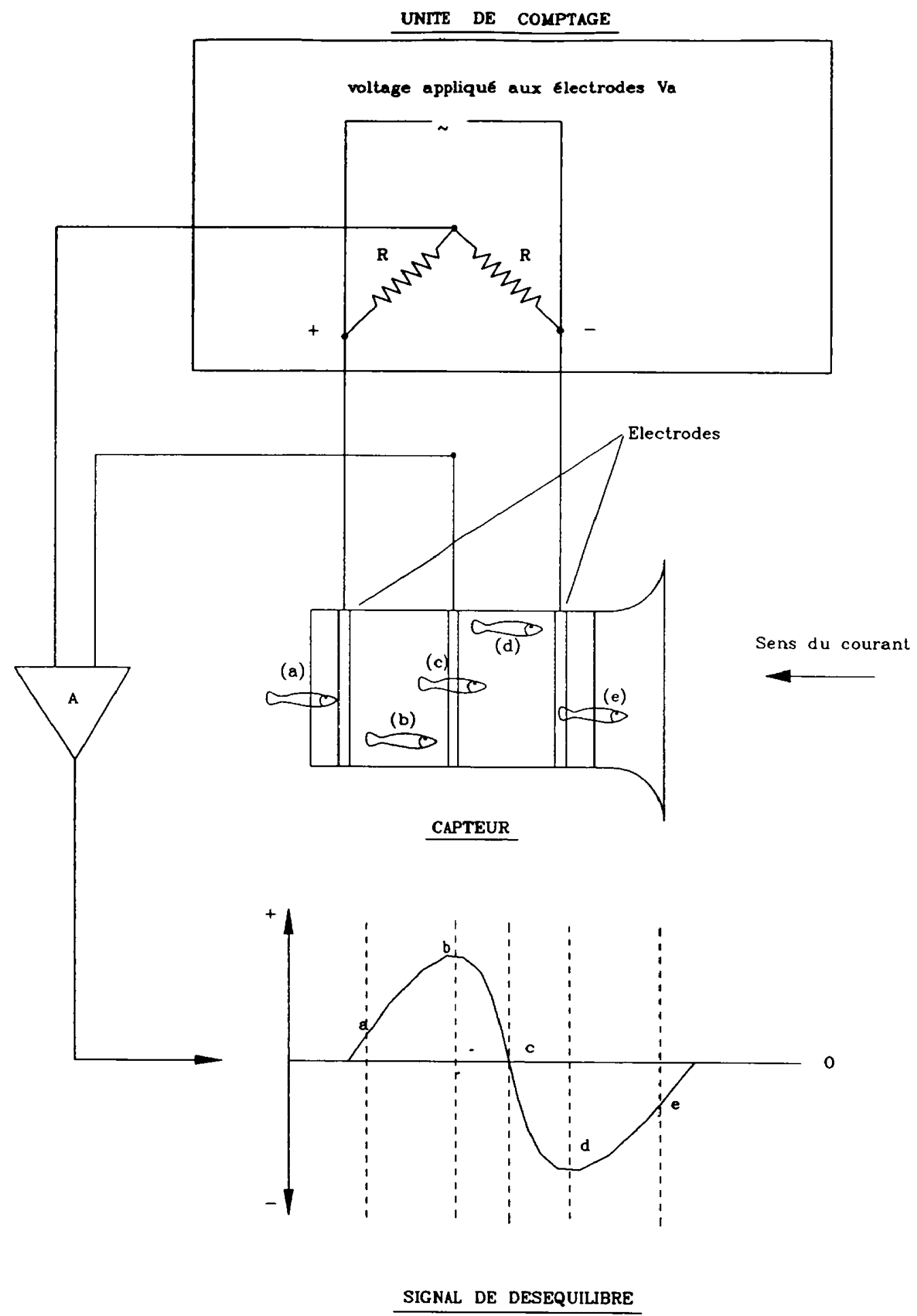

Figure 2 : Principe de fonctionnement d'un compteur automatique à résistivité.

Figure 2 : Resistivity automatic counter. Principle of the operation. 
Le passage du poisson dans le champ des électrodes induit un signal de déséquilibre du pont qui est analysé pour déterminer le sens de passage du poisson et en classifier la taille (séparation en deux classes de taille en général). Un rattrapage automatique de la sensibilité du compteur permet de maintenir constantes les tailles de poissons discriminées lorsque la conductivité de l'eau varie.

Un circuit détecteur de niveau interrompt le fonctionnement du compteur dès que la hauteur d'eau sur les électrodes est insuffisante de façon à éviter les faux comptages induits par les ondulations de la surface libre.

L'utilisation d'un tel type de compteur est courante en Grande-Bretagne, où il a fait l'objet d'un programme de recherche intense dans les années 70 de la part du Ministère de l'Agriculture (MAFF) (BUSSEL, 1978 ; DUNKLEY et SHELTON, 1991). Plusieurs modèles sont commercialisés : compteurs du NSHEB et compteur Sharkey de Marine Electrics. Un compteur a également été développé en France par les Ets Fron, en collaboration avec le CEMAGREF et I'INRA (BONNEFOIS, 1988 ; GOSSET, 1986), ceci pour faciliter la maintenance et réduire les coûts d'investissement et de fonctionnement par rapport à du matériel étranger.

La géométrie du capteur peut être variable suivant le site. Lorsque le compteur est installé dans une passe à poissons, on choisit généralement des électrodes circulaires installées dans un tube implanté dans un bassin de la passe. Le diamètre du tube est fonction de la configuration de la passe et de son débit ainsi que de la taille minimale des poissons à compter, les diamètres couramment utilisés varient généralement de $0.30 \mathrm{~m}$ à $0.50 \mathrm{~m}$.

Le compteur est généralement couplé à un enregistreur d'événements permettant de dater les passages, ceci autant pour l'importance biologique que revêt cette information que pour pouvoir éliminer les éventuels artefacts de comptage.

La fiabilité du comptage (qui peut être supérieure à $80 \%$ ) dépend avant tout de la qualité de l'implantation du capteur et de la fréquence de surveillance :

- il convient d'éviter, dans le capteur, les turbulences excessives et l'entraînement d'air susceptible d'induire des faux comptages. On doit y assurer par ailleurs des vitesses de courant suffisamment fortes (supérieures à $1 \mathrm{~m} / \mathrm{s}$ ) pour éviter que le poisson ne stationne entre les électrodes.

- un suivi fréquent (de préférence quotidien et au minimum hebdomadaire) doit être assuré par un personnel qualifié. Celui-ci, sans posséder obligatoirement des connaissances en électronique, doit absolument avoir suivi une formation minimale devant lui permettre de détecter les principales causes d'anomalies ou de dysfonctionnement : dégradation du capteur, défaut d'alimentation, faux comptages induits par des entraînements d'air ou des vortex, pannes dans le circuit logique...

L'avantage du compteur réside dans ses faibles coûts d'investissement (de l'ordre de $20000 \mathrm{~F}$ pour le compteur, entre $5000 \mathrm{~F}$ et $10000 \mathrm{~F}$ suivant le site pour la fabrication et l'installation du capteur) et de maintenance (besoin en personnel très réduit).

L'inconvénient majeur, inhérent à son principe, est l'absence de discrimination des espèces autrement que par leur taille. Son utilisation est pratiquement limitée aux cours d'eau à grands salmonidés migrateurs où l'on peut espérer compter et distinguer saumons, truites de mer et truites uniquement sur le critère de taille. Encore faut-il s'assurer que sur ces cours d'eaux, il n'y a pas de risque de perturbation des comptages par d'autres espèces migratrices. A ce titre, la lamproie s'avère très perturbatrice du fait de sa propension à se "ventouser" sur les capteurs.

\subsection{Comptage visuel ou par enregistrement vidéo}

Le comptage visuel est une technique de comptage en continu sans manipulation des poissons qui permet un dénombrement en s'affranchissant des inconvénients majeurs du piégeage. Il consiste à faire passer les poissons dans une zone où ils soient suffisamment visibles pour être identifiables et dénombrables. Deux techniques de visualisation sont utilisées : visualisation externe "par-dessus" où l'on force le poisson à 
franchir une zone de couleur claire à faible profondeur sur laquelle il se détache en "ombre chinoise" et visualisation latérale où l'on oblige le poisson à passer devant une baie vitrée verticale. Cette dernière solution offre, par rapport à la première, l'avantage de permettre la reconnaissance de la majorité des espèces dans la mesure où les critères morphologiques de distinction sont beaucoup plus apparents sur une vue latérale que sur une vue "pardessus".

Dans les passes à bassins ou à ralentisseurs, la visualisation externe est réalisée grâce à l'installation d'une plaque horizontale en amont d'un dispositif de guidage des poissons (grille, échancrure d'une cloison) immergée sous une profondeur d'eau ne dépassant pas $0.30 \mathrm{~m}$. Pour les écluses à poissons l'installation de la plaque se fait directement sur la vanne de régulation amont. Dans les ascenseurs à poissons à cuve de petite taille (épaisseur d'eau ne dépassant pas $0.40 \mathrm{~m}$ ), la visualisation se fait directement au-dessus de la cuve dont le fond est peint d'une couleur claire.

Pour la visualisation latérale on aménage une restriction de passage (à l'aide de grilles déflectrices ou d'un rétrécissement de la structure) au droit de la baie vitrée forçant ainsi le poisson à passer à proximité. Une largeur minimale de passage de 40 à $50 \mathrm{~cm}$ est en général adoptée afin de ne pas perturber les poissons. Elle peut être réglable $(0.30 \mathrm{~m}$ à $1 \mathrm{~m}$ ) pour s'adapter aux variations de turbidité de l'eau. Une plaque de visualisation, de couleur claire, en général carroyée de façon à avoir une idée de la taille des poissons, est placée au droit de la vitre (Fig. 3).

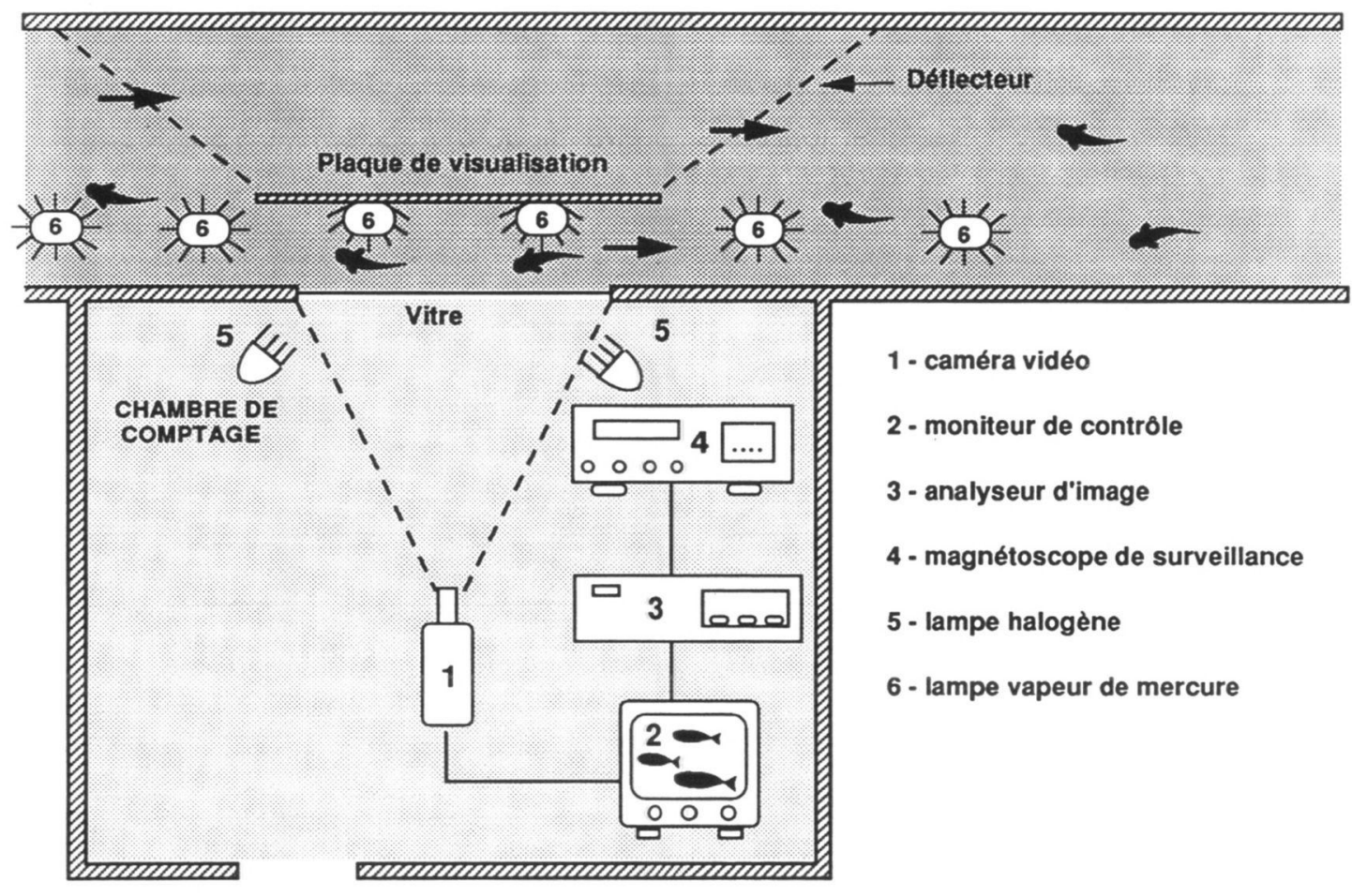

SCHEMA DE PRINCIPE (Vu en plan) DU DISPOSITIF DE COMPTAGE VIDEO AUTOMATIQUE "CERBERE"

Figure 3 : Vue schématique du dispositif de comptage vidéo CERBERE.

Figure 3 : Schematic plan view of the automatic video fish passage recording system "CERBERE". 
Pour que le comptage se fasse dans de bonnes conditions, il est nécessaire que les poissons passent devant le dispositif de visualisation avec une vitesse compatible avec les possibilités de détermination des espèces en temps réel, qu'il n'y ait pas d'arrêt et le moins possible d'allers et retours des poissons devant le dispositif. A cet effet, on maintient dans la zone d'observation une vitesse d'écoulement de l'ordre de $1 \mathrm{~m} / \mathrm{s}$ à $1.5 \mathrm{~m} / \mathrm{s}$ (variable suivant les espèces et les sites), et on implante de préférence le dispositif le plus en amont possible de la passe de façon à s'affranchir des allers et retours fréquents de certaines espèces migratrices dans les passes. Un éclairage de la zone de visualisation s'impose pour maintenir des conditions optimales de visibilité quelle que soit l'heure de la journée.

II convient par ailleurs d'éviter avec soin la création, au droit de la vitre, de zones de décollement dans lesquelles certains individus ou espèces auront tendance à stationner.

Le comptage peut être réalisé en temps réel : il nécessite alors la présence d'un observateur permanent. Cette solution, très lourde en temps, se justifie pour les très gros ouvrages (rivière Columbia, USA) où les passages de poissons sont continus. Elle présente en outre l'inconvénient de ne pouvoir effectuer sur les passages aucune analyse différée de certains paramètres non saisissables en temps réel (taille des poissons, comportement des diverses espèces, vitesse de passage).

Les progrès récents en matière de technique vidéo - en particulier dans la technique de surveillance - ont permis d'automatiser dans une certaine mesure et d'alléger les opérations de comptage visuel réalisées par des observateurs in situ. Plusieurs types de solutions sont adoptés à l'heure actuelle en France.

Pour le contrôle des ascenseurs à salmonidés ayant une cuve à faible capacité (profondeur d'eau de l'ordre de $0.20 \mathrm{~m}$ dans le fond de la cuve en phase de remontée), l'automatisation est facilitée du fait du caractère discontinu des passages. On utilise une caméra fixée à l'aplomb de la cuve et l'on met automatiquement en route pendant quelques secondes un magnétoscope lors de la remontée. Une installation de ce type fonctionne à l'ascenseur à saumons de Poutès sur la rivière Allier depuis 1986. Elle a permis un comptage 24h/24 (12 remontées par jour) avec un travail de dépouillement n'excédant pas une heure par mois et une fiabilité supérieure à $95 \%$.

Une méthode similaire a été utilisée ponctuellement sur l'écluse à poissons de Soeix sur le Gave d'Aspe : une plaque de visualisation a été installée au niveau de la sortie, sur la vanne amont. Cette méthode nécessite cependant de filmer en continu lors de la phase de remplissage et de sortie et les durées de dépouillement se révèlent beaucoup plus longues que dans le cas des ascenseurs. façons :

Pour les vitres immergées, où les passages sont continus, on peut procéder de deux

- enregistrement vidéo en continu à vitesse réduite et dépouillement des bandes vidéo en accéléré grâce à un magnétoscope à temps échelonné. Les enregistrements s'effectuent de telle sorte qu'une bande vidéo de trois heures dure $24 \mathrm{~h}$ ou $48 \mathrm{~h}$, soit une réduction de vitesse d'enregistrement dans un rapport de $1 / 8$ à 1/16. Le dépouillement devient cependant rapidement une opération très longue et fastidieuse : le dépouillement d'une bande de 24 heures peut demander 3.5 à 5 heures dès que le nombre des poissons devient important, du fait des nécessités de retour en arrière très fréquentes pour la détermination des espèces,

- automatisation de l'enregistrement avec détection des poissons lors de leur arrivée dans la zone de comptage, de façon à n'enregistrer que les épisodes de passage par enclenchement automatique d'un magnétoscope. La Direction des Etudes et Recherches d'EDF (TRAVADE, 1990) a mis au point un système utilisant la détection du poisson au niveau de la vitre elle-même par un traitement sommaire de l'image vidéo (Fig. 3). Le système, dénommé CERBERE, comprend une caméra vidéo filmant la vitre en permanence reliée à un magnétoscope à temps échelonné (type Panasonic NV 8051 ou AG 6720) ayant la particularité de tourner à vitesse réduite ralentie ( $480 \mathrm{~h}$ pour une bande classique de $3 \mathrm{~h}$ ) et de se mettre en enregistrement à vitesse normale sur une alarme extérieure. Cette alarme est générée par un analyseur d'image (matériel FOR-A SVS660) lors de l'intrusion d'un poisson dans l'image vidéo. Le système permet donc d'enregistrer uniquement les séquences pendant lesquelles un poisson est présent devant la vitre tant à 
la montée qu'à la descente. Un certain nombre de réglages de sensibilité permettent d'étalonner le dispositif en fonction des caractéristiques du site et des poissons.

La détermination des espèces et leur dénombrement s'effectuent en visionnant les enregistrements a posteriori. Ce système a été utilisé avec succès pour le comptage $24 \mathrm{~h} / 24$ dans les ouvrages situés sur les grands cours d'eau : Tuilières et Mauzac sur la Dordogne, Golfech et le Bazacle sur la Garonne. II a permis de comptabiliser avec un seul observateur jusqu'à plusieurs milliers de poissons par jour appartenant à plus de 20 espèces différentes en donnant des indications précieuses sur les rythmes migratoires.

Les durées théoriques de dépouillement vont de $10 \mathrm{mn}$ par $24 \mathrm{~h}$ de surveillance pour moins de 10 poissons/jour jusqu'à $7 \mathrm{~h}$ par $24 \mathrm{~h}$ pour un comptage de 5000 poissons/jour. En réalité ces valeurs sont fonction des conditions de milieu (visibilité, déclenchements parasites par des corps dérivants) et surtout des espèces comptabilisées (facilité de distinction des espèces, vitesse de passage, passages isolés ou en bancs, allers et retours, stabulation devant la vitre). A l'expérience des comptages in situ, il ressort qu'en moyenne les durées de dépouillement sont de l'ordre de $4 \%$ à $10 \%$ du temps réel de surveillance (soit $1 \mathrm{~h}$ à $2.5 \mathrm{~h}$ par $24 \mathrm{~h}$ de suivi) pour les faibles passages (inférieurs à 400 poissons/jour) et de l'ordre de $15 \%$ à $20 \%$ du temps réel (soit $3.5 \mathrm{~h}$ à $5 \mathrm{~h}$ par $24 \mathrm{~h}$ de suivi) pour les passages nombreux ( 3000 à 5000 poissons/jour).

La fiabilité de détection du poisson devant la vitre dépend de divers paramètres de milieu (éclairage, turbidité de l'eau pour laquelle un minimum de $0.70 \mathrm{~m}$ au disque de secchi est requise) et des espèces de poissons considérées (en fonction de leur taille, de leur couleur, de leur vitesse et de leur profondeur de nage). En moyenne, dans de bonnes conditions de visibilité, la fiabilité de détection est excellente $(90 \%$ à $100 \%)$ pour les salmonidés, l'alose, et les cyprinidés de taille supérieure à $25 \mathrm{~cm}$, bonne $(70 \%$ à $90 \%)$ pour les lamproies, barbeaux et cyprinidés de taille comprise entre $10 \mathrm{~cm}$ et $25 \mathrm{~cm}$, et moyenne (50\% à $70 \%$ ) pour l'anguille et les poissons de taille inférieure à $10 \mathrm{~cm}$.

Les caractéristiques de l'éclairage de la zone de visualisation sont particulièrement importantes vis-à-vis du fonctionnement du système CERBERE dans la mesure où elles agissent à la fois sur la capacité de détection des poissons par le système et sur le comportement des poissons. II est nécessaire d'éclairer simultanément au-dessus de la surface de l'eau et à travers la baie vitrée. L'éclairage de surface, suffisamment puissant au-dessus de la vitre, (environ $2000 \mathrm{~W}$ en lampes halogène, $500 \mathrm{~W}$ en lampes à vapeur de mercure) doit s'étendre en intensité dégressive à plusieurs mètres en amont et en aval de la vitre pour éviter des perturbations de comportement (stagnation devant la vitre, demitours), surtout au cours de la nuit.

Une surveillance régulière (quotidienne à trihebdomadaire) est absolument nécessaire pour changer les bandes vidéo, contrôler les réglages du dispositif, nettoyer la vitre de visualisation et la zone de passage des poissons.

Le coût d'une installation de comptage visuel comprend la station de comptage ellemême (local, dispositif de guidage pour les poissons, éclairage) et le système vidéo. Le coût de la station est variable suivant les caractéristiques du site et la sophistication de l'installation : de $100000 \mathrm{~F}$ à $200000 \mathrm{~F}$. Le coût d'un système vidéo permettant un contrôle en continu et un dépouillement simultané (minimum de 2 magnétoscopes, 1 caméra vidéo, deux moniteurs) est le suivant :

- de $20000 \mathrm{~F}$ à $40000 \mathrm{~F}$ pour un système de surveillance d'ascenseur à poissons (possibilité d'utilisation de magnétoscopes "grand public"),

- de $60000 \mathrm{~F}$ à $80000 \mathrm{~F}$ pour un système de suivi "en continu" mettant en oeuvre des magnétoscopes type "surveillance" sans dispositif d'enregistrement automatique,

- de $100000 \mathrm{~F}$ à $120000 \mathrm{~F}$ pour le système "CERBERE" complet comprenant l'analyseur d'image.

Les avantages du comptage visuel sont les suivants : absence de manipulation des poissons, possibilité de comptage des espèces difficiles à piéger (alose), charge en personnel moins lourde que pour le piégeage, précision extrême sur la détermination des rythmes de migrations et sur le comportement de l'ensemble des espèces. 
Les inconvénients sont l'impossibilité de comptage par forte turbidité de l'eau et la difficultés de détermination de certaines espèces. Pour les systèmes automatisés (CERBERE, par exemple) on se heurte également à l'efficacité partielle de détection de certaines espèces, et à la sensibilité du système aux corps dérivants (herbiers, en particulier) qui provoquent des déclenchements intempestifs de l'enregistrement vidéo. Dans les zones où de nombreuses espèces sont présentes une grande partie de l'année, le travail de dépouillement des bandes vidéo reste relativement lourd et fastidieux.

\subsection{Comptage et reconnaissance automatique par analyse d'image}

Devant la lourdeur des opérations de dépouillement de comptage visuel dès que le stock de migrateurs devient important et suite aux progrès récents dans le domaine de la vision assistée par ordinateur, il était tentant de chercher à aller plus loin dans la mise en place de systèmes automatiques. Des recherches ont été récemment entreprises par le CEMAGREF, EDF et le laboratoire d'électronique de l'ENSEEIHT à Toulouse (LARINIER et TRAVADE, 1992) dans le but de mettre au point un système automatique reposant sur une machine de vision. Celle-ci devra reconnaître et compter un certain nombre d'espèces ou groupe d'espèces, en priorité les grands salmonidés migrateurs (saumon, truite de mer), les aloses, les lamproies, les anguilles et éventuellement les barbeaux et les brèmes audessus d'une certaine taille. II devra être capable de conserver une trace (date et heure de passage, image ou contour) pour certaines espèces particulières.

Le principe du système automatique de reconnaissance et comptage est de numériser chaque image vidéo, de la segmenter pour en isoler les objets, d'en mesurer un certain nombre de caractéristiques de forme, d'identifier ces objets (reconnaissance de l'espèce s'il s'agit d'un poisson) et de les compter par un suivi dynamique.

L'amélioration des conditions d'acquisition des images a été le problème crucial du début de l'étude puisqu'il s'est révélé impossible, sur des images prises dans les conditions actuelles d'éclairage des stations de contrôle, d'obtenir des résultats qui soient exploitables après traitement : problème de non-uniformité de l'éclairage, de contraste insuffisant, d'effet de flou sur certaines parties essentielles du poisson comme la nageoire caudale empêchant d'isoler du fond les formes significatives.

L'utilisation d'une caméra avec obturateur électronique (temps de pose de l'ordre de 1 à 4 millisecondes) élimine l'effet de flou sur les contours. Le recours à un rétroéclairage a permis par ailleurs d'obtenir des silhouettes se détachant bien du fond; il a cependant l'inconvénient d'éliminer toute information sur la texture.

Dans l'état actuel d'avancement de l'étude, la discrimination des espèces-cibles par reconnaissance de forme (images figées) apparaît réalisable. L'application de ces résultats à la reconnaissance et au comptage en temps réel (suivi dynamique du poisson sur une séquence d'image) fait l'objet d'une poursuite des recherches. II est encore difficile de se prononcer sur l'échéance de mise en service opérationnelle d'un tel système.

\section{ESTIMATION DE L'EFFICACITÉ RÉELLE D'UN OUVRAGE DE FONCTIONNEMENT}

L'efficacité réelle d'une passe s'évalue en termes de proportion de migrateurs présents au pied de l'obstacle empruntant cette passe et en termes de retard à la migration. Deux types de méthodes sont utilisables dans cette approche.

\subsection{Méthodes quantitatives ou statistiques}

Une première méthode, directe, consiste à mesurer ou à estimer la taille de la population à l'aval de l'ouvrage et à la comparer aux passages déterminés par comptage. La taille de la population peut être appréhendée par comptage dans un piège ou un dispositif de franchissement situé à l'aval ou par suivi quantitatif de pêcheries.

Une autre méthode, indirecte, consiste à marquer un certain nombre de poissons à l'aval de l'ouvrage et à dénombrer les poissons marqués empruntant la passe. L'efficacité de l'ouvrage est donnée par la formule: 


$$
E=100\left(N_{p} / C N_{m}\right)
$$

$E$ : efficacité de la passe en \%.

$N_{p}$ : nombre de poissons marqués passant dans l'ouvrage.

$\mathrm{N}_{\mathrm{m}}$ : nombre de poissons marqués à l'aval.

$\mathrm{C}$ : coefficient $(0<\mathrm{C} \leq 1)$ exprimant l'influence du marquage.

La population de migrateurs présents au pied de l'ouvrage peut être estimée en effectuant un comptage de la totalité des passages:

$$
\mathrm{N}_{\mathrm{e}}=\mathrm{CNN}_{\mathrm{m}} / \mathrm{N}_{\mathrm{p}}
$$

$\mathrm{N}_{e}$ : population estimée à l'aval de l'ouvrage.

$N$ : nombre total d'individus ayant transité dans la passe.

En pratique, toute la difficulté consiste à estimer l'effet du marquage (coefficient $C$ ) qui peut être négligeable pour les espèces résistantes (salmonidés par exemple) mais important pour certaines espèces sensibles (alose par exemple) chez lesquelles le marquage peut induire des modifications de comportement migratoire, voire des mortalités significatives.

\subsection{Méthodes comportementales}

Ces méthodes destinées à mettre en évidence les facteurs influant sur l'efficacité des ouvrages (localisation des entrées, débit dans l'ouvrage, effet de certains paramètres de milieu) consistent à suivre directement les déplacements et le comportement de quelques individus à l'approche des ouvrages. On utilise à cet effet des techniques télémétriques qui reposent sur la détection à distance, à l'aide de récepteurs appropriés, de la position d'un émetteur implanté sur le poisson. Deux techniques de repérage télémétrique des poissons sont utilisées dans le monde :

- la télémétrie ultrasonique utilisant des émetteurs dans la bande de fréquence 20-100 kHz (STATSKO et PINCOCK, 1977). Elle présente l'avantage de pouvoir suivre des poissons dans des eaux de conductivité quelconque (eau douce et eau de mer) mais a comme inconvénients d'être perturbée par tous les bruits ambiants (écoulements turbulents, fonctionnement des turbines...), de nécessiter un repérage à l'aide d'hydrophones immergés, et d'être "gourmande" en énergie d'émission, d'où la faible durée de vie des émetteurs (quelques dizaines de jours au maximum),

- la radiotélémétrie utilisant des émetteurs radio dans la bande de fréquence 20-180 MHz (MCLEAVE et al., 1978 ; SOLOMON, 1982 ; TRAVADE et al., 1989). Elle présente l'avantage de pouvoir utiliser des antennes aériennes ou immergées, à des distances relativement importantes (jusqu'à $2 \mathrm{~km}$ dans les meilleures conditions), de ne pas être perturbée par les bruits ambiants du cours d'eau ou des usines hydroélectriques, et d'être peu gourmande en énergie, d'où la possibilité d'utilisation d'émetteurs de petite taille sur des durées de plusieurs mois. Elle est cependant strictement limitée aux eaux douces du fait de la très forte absorption des ondes radio par l'eau dès que la conductivité dépasse 300 à $400 \mu \mathrm{S} / \mathrm{cm}$.

De ce fait, c'est essentiellement la radiotélémétrie (ou "radiopistage") qui est utilisée pour le suivi des migrateurs en rivière (conductivité inférieure à $400 \mu \mathrm{S} / \mathrm{cm}$ ), la télémétrie sonique étant réservée aux études en estuaire et en mer.

L'émetteur miniaturisé (poids de $2 \mathrm{~g}$ à $25 \mathrm{~g}$ suivant la taille des migrateurs concernés) est implanté sur le poisson de façon interne (estomac ou cavité générale) ou externe (la plupart du temps sur la musculature dorsale au droit de la nageoire dorsale). Pour les grands migrateurs adultes ne se nourrissant pas à la remontée (saumon, alose), l'implantation dans l'estomac est la plus facile à réaliser et la moins perturbante pour le poisson. Après anesthésie du poisson, la marque est poussée au fond de l'estomac à l'aide d'une tube applicateur. L'antenne est soit passée entre les arcs branchiaux et rabattue le long du ventre (saumon, truite de mer), soit pour les espèces plus fragiles (alose), laissée à l'angle du maxillaire.

Le plus grand soin doit être apporté à la manipulation du poisson, tant au niveau de la capture et de l'anesthésie que de l'implantation de l'émetteur, de façon à éviter de perturber son comportement. 
Le suivi des poissons marqués peut revêtir de multiples aspects suivant la taille des cours d'eau, la nature de l'étude ou le niveau de précision requis :

- pistage manuel : le poisson est repéré à l'aide d'antennes aériennes portables directionnelles (type boucle, Adcock) ou non directionnelles (type fouet) qui peuvent être manipulées à pied ou à bord de véhicules divers (voiture, bateau, avion). Pour un repérage de grande précision (quelques $\mathrm{cm}$ ), il est possible d'utiliser des antennes-boucles immergeables fixées à l'extrémité d'une perche. Dans les cours d'eau de grande largeur, le positionnement précis du poisson fait appel à la biangulation ou à la triangulation, consistant à relever les azimuts de l'émetteur à l'aide de deux ou trois récepteurs à poste fixe, la position du poisson étant donnée par l'intersection des azimuts,

- repérage automatique : la détection automatique de présence des poissons dans une zone est réalisable à l'aide de dispositifs d'enregistrements graphiques ou à mémoire (possibilité de traitement direct des résultats par informatique) annexés au récepteur. Suivant la taille de la zone à surveiller, il est possible d'utiliser des antennes aériennes (couverture importante) ou des antennes immergées (couverture restreinte, comme le bassin d'une passe à poissons par exemple). La présence des poissons dans la zone concernée est enregistrée en fonction du temps. La combinaison judicieuse de plusieurs postes d'enregistrement sur un site, permet de suivre automatiquement, par recoupement, les déplacements de poissons. Ces stations nécessitent l'emploi de récepteurs à scanner (défilement automatique des fréquences de chacun des poissons) ou de récepteurs travaillant en parallèle sur plusieurs canaux.

Le matériel utilisé dans les études réalisées récemment en France (TRAVADE et al., 1989) provient des USA (marque Advanced Telemetry Systems). II n'existe pas à l'heure actuelle de fabricant français pour ce type de matériel.

La mise en oeuvre de cette technique est relativement coûteuse tant en matériel qu'en main d'oeuvre : le coût de l'équipement est d'environ $15000 \mathrm{~F}$ pour un récepteur portable, $15000 \mathrm{~F}$ pour un enregistreur graphique, et de 1000 à $1500 \mathrm{~F}$ par émetteur, ce dernier étant considéré comme perdu après étude. Le personnel nécessaire à l'étude est estimé à 1 à 2 personnes pour une petite étude réalisée avec des systèmes d'enregistrement des déplacements de poissons et 3 à 10 personnes pour une étude de plus grande ampleur nécessitant un repérage manuel des poissons.

Quoique coûteuse, cette technique dont la spécificité est de pouvoir "observer" en direct le comportement des poissons dans les dispositifs de franchissement et à leur approche, s'avère irremplaçable pour obtenir rapidement des informations sur leur fonctionnalité et pour permettre d'affiner certains critères de conception particuliers.

\section{BIBLIOGRAPHIE}

BELL M.C., 1986. Fisheries handbook of engineering requirements and biological criteria. Fish. Eng. Res. Prog., U.S. Army Corps of Eng., North Pacific Div., Portland, Oregon, $290 \mathrm{p}$.

BONNEFOIS P., 1988. Le compteur à poissons type "FRON" au sein des techniques de comptage, CEMAGREF, $110 \mathrm{p}$.

BUSSEL R.B., 1978. Fish counting stations (notes for guidance in their design and use). Dept. of the Envir., Londres, $97 \mathrm{p}$.

CLAY C.H., 1961. Design of fishways and other fish facilities. Dept. of Fisheries, Ottawa, Canada, $301 \mathrm{p}$.

DUNKLEY D.A., SHELTON G.J., 1991. Recent developments in automatic fish counters for salmon rivers. Int. Council for the Exploration of the Sea, CM 1991/M, 27, 5 p.

GOSSET C., 1986. Présentation et essais d'un compteur à résistivité pour poissons. Bull. Fr. Pisc., 303 : 141-151.

LARINIER M., TRAVADE F., 1992. Utilisation des dispositifs de franchissement pour le suivi des stocks et la gestion des populations de poissons migrateurs. Colloque francoquébécois sur l'utilisation des technologies modernes à la gestion des poissons dulcicoles et amphihalins, Montréal, $12 \mathrm{p}$. 
MCLEAVE J.D., POWER J.H., ROMMEL S.A., 1978. Use of radio telemetry for studying upriver migration of adult atlantic salmon (Salmo salar). J. Fish. Biol., 12 : 549-558.

SOLOMON D.J., 1982. Tracking fish with radio tags. Symp. Zool. Soc. Lond., 49 : 95-105.

STATSKO A.B., PINCOCK D.G., 1977. Review of underwater biotelemetry with emphasis on ultrasonic techniques. J. Fish. Res. Bd. Can., 34 : 1261-1285.

TRAVADE F., 1990. Monitoring techniques for fish passes recently used in France. Proc. Int. Symp. on fishways, Gifu, Japan, 119-126.

TRAVADE F., BOMASSI P., BACH J.M., BRUGEL C., STEINBACK P., LUQUET J.F., PUSTELNICK G., 1989. Use of radiotracking in France for recent studies concerning the EDF fishway program. Hydroécol. Appl., 1/2 : 33-51. 


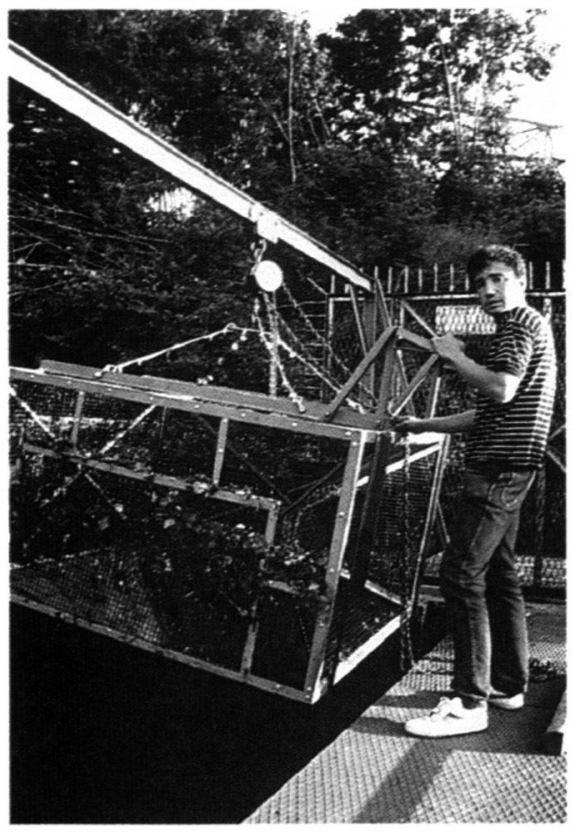

Photo 1 : Nasse implantée à l'amont d'une passe à poissons.

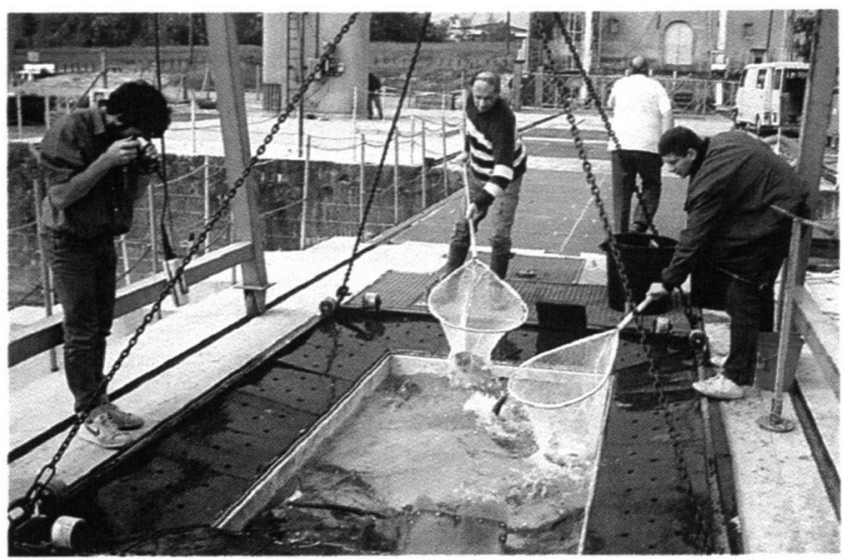

Photo 2 : Dispositif de comptage par le relevage du fond mobile d'un piège.

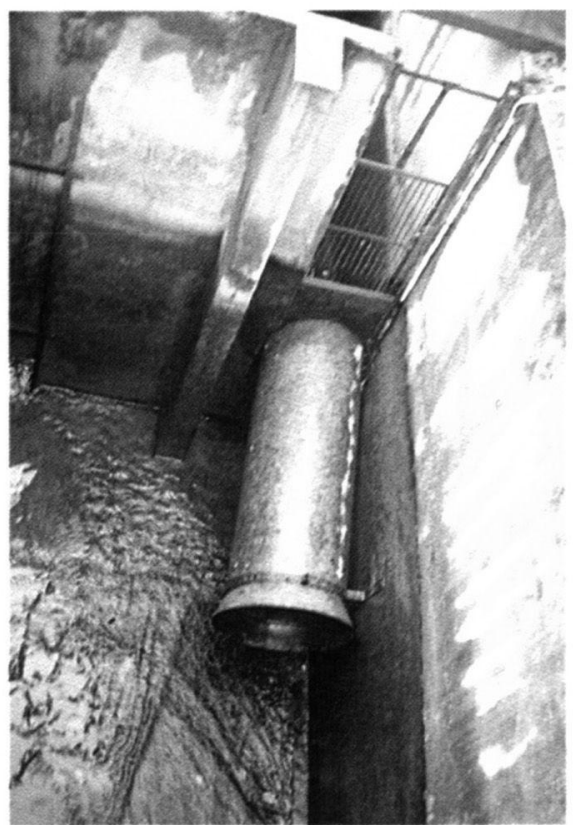

Photo 3 : Capteur pour compteur automatique à résistivité implanté dans l'échancrure d'une passe.
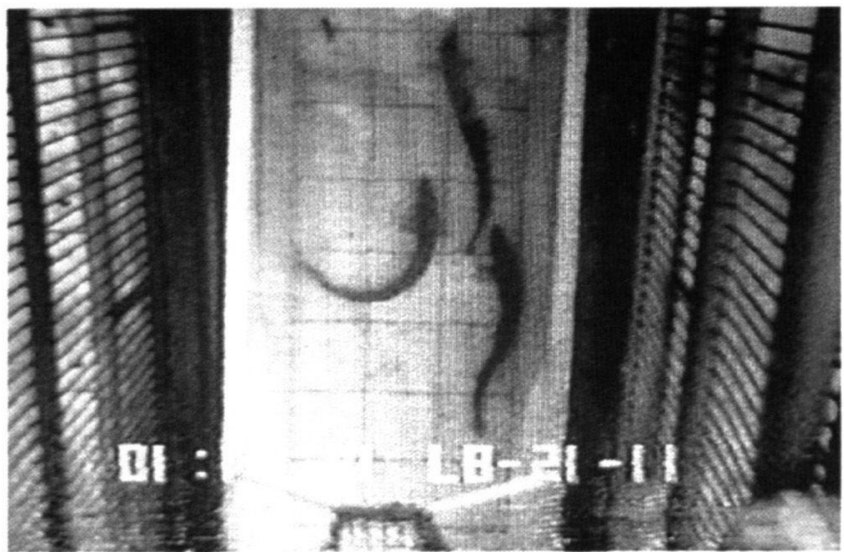

Photo 4 : Contrôle vidéo des poissons dans un ascenseur. 


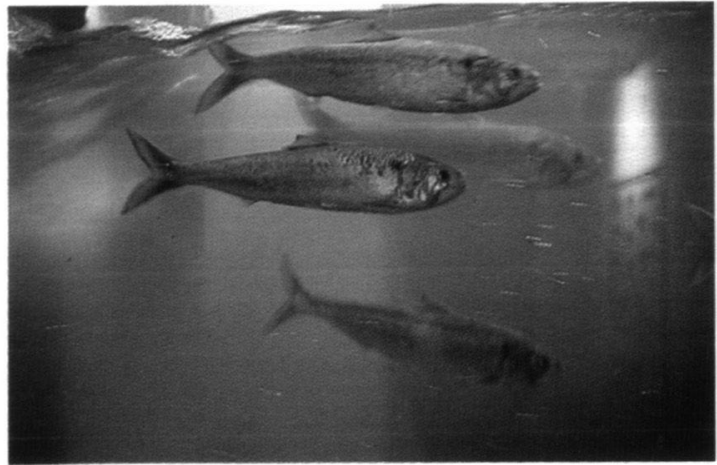

Photo 5 : Visualisation d'aloses derrière une vitre de comptage.

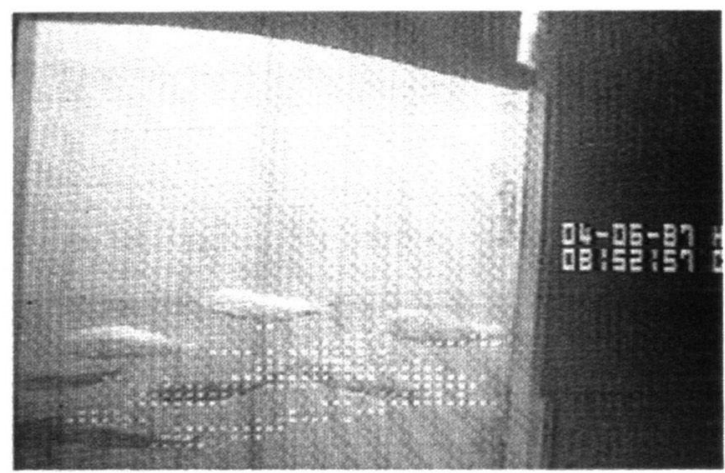

Photo 7 : Enregistrement des passages d'aloses par le système vidéo automatique "CERBERE".

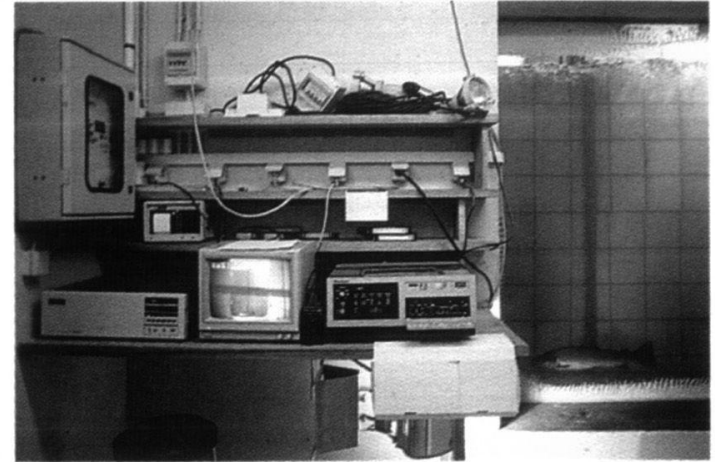

Photo 6 : Système vidéo de comptage automatique "CERBERE" (passe à poissons de Golfech sur la Garonne).

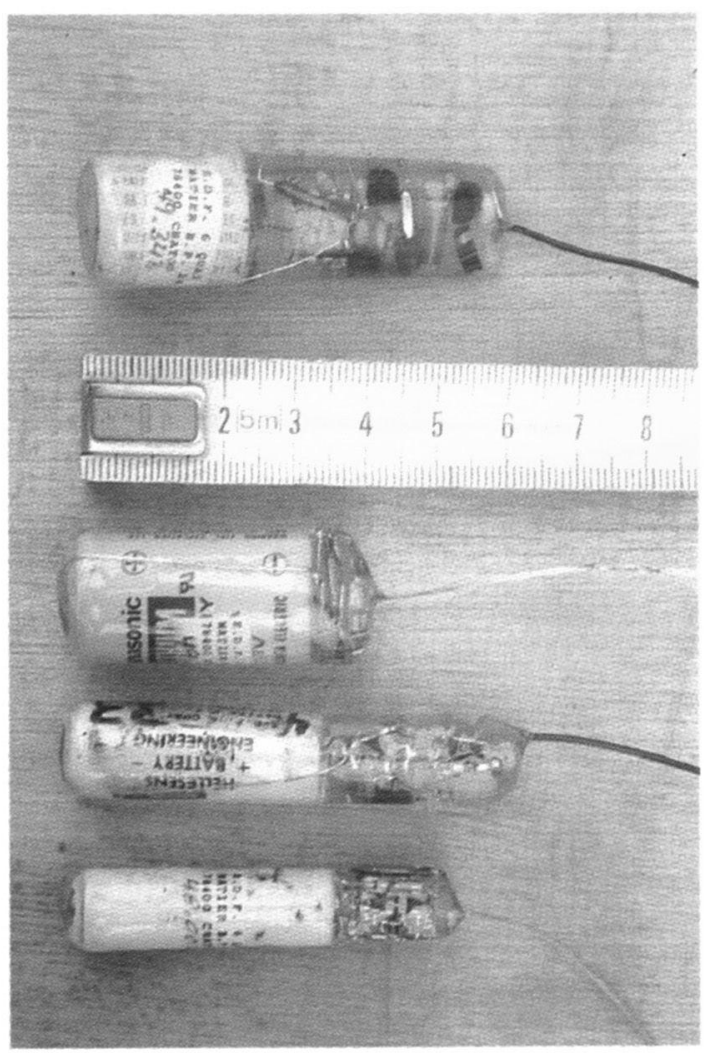

Photo 8 : Emetteurs miniaturisés pour le radiopistage. 